\title{
The category effect in visual search: Is faster mixed-category search due to the priming of category information?
}

\author{
BELINDA M. BOYD-WILSON and MURRAY J. WHITE \\ Victoria University of Wellington, Wellington, New Zealand
}

\begin{abstract}
Search for a target surrounded by members of an opposite class (e.g., a digit among letters) is, typically, faster than search for a target surrounded by members of the same class. A promising explanation for this effect is related to priming processes. If presentation of an item primes both its category and its identity, that item, subsequently appearing amidst opposite-category items, will be distinct. When a previously specified item is presented amidst same-class items, however, category information contained in all will be primed. Field items will attract attention, slowing down search. To test this hypothesis, two stimuli were presented in succession prior to a search display that contained three stimuli. Either of the two might be the target in the display. The stimuli were sketches of faces. When the two faces were of the same sex, search was faster if the categories were mixed. When the two faces were of different sex, this category effect was reversed: Search was faster when the categories were the same.
\end{abstract}

During the past two decades, researchers have produced an effect whose cause they have been unable to agree upon. It is that a specified item is found more quickly when surrounded by items from a different class (e.g., a letter among digits) than when surrounded by items from the same class. This has been dubbed the category effect, and it has been observed for letters and digits (e.g., Jonides \& Gleitman, 1972) and for words that belong to different semantic categories (Henderson \& Chard, 1978; Karlin \& Bower, 1976).

Explanations of the category effect have varied considerably. It has been attributed to a greater ease of discrimination that owes to the presence of distinctive physical features in a mixed- or between-category condition (see, e.g., Duncan, 1983; Krueger, 1984). But it is doubtful whether digits as a class contain features that distinguish them from letters (Deutsch, 1977). In any case, the featural explanation falters in the light of the semantic category effects that have been observed. The lack of a category effect following the matching of stimuli between categories (Krueger, 1984; White, 1977) may have resulted from confusion in between-category but not within-category search (Dixon \& Shedden, 1987).

The category effect has also been attributed by some to categorization's being easier or faster than identification (Jonides \& Gleitman, 1972), but other researchers have found the opposite to be true (Dick, 1971; Nickerson, 1973; White, 1977). The category effect has been

This research was carried out by Belinda Boyd-Wilson for an Honors thesis and was supervised by Murray White. Requests for reprints should be sent to Murray White, Psychology Department, Victoria University of Wellington, P.O. Box 600, Wellington, New Zealand. attributed to localization processes; in a between-category condition, a target is localized because of its category difference, and field items need not be localized (Butler, 1980; Jonides \& Gleitman, 1976). But category effects have been found in a serial task when localization processes were patently not involved (Fletcher, 1981).

Again, the category effect has been linked with automaticity. It has been suggested that field items in betweencategory search can be processed in parallel because of their category difference (Duncan, 1980; Henderson \& Chard, 1978). This account implies that categorization precedes identification, an implication already called into question.

Yet another explanation for the category effect, which can be traced to the suggestions of Deutsch (1977) and Kahneman and Treisman (1984), has to do with priming. Striking perceptual priming effects have been recorded (Posner \& Snyder, 1975), but evidence suggests that the class or category of stimuli can be implicitly primed too. For example, Palmer and Jonides (1988) required subjects to judge whether a probe word, presented after a prime word, was a member of a memory set of several words presented prior to the prime. Primes never appeared in the memory sets. These experimenters discovered that an appropriate response to the probe was markedly speeded when the stimuli in a trial all belonged to a distinct category (boys' and girls' names) rather than an arbitrary category, suggesting that category information contained within primes affected responses. The category effect can thus be explained in the following manner: A subject, alert to category differences because of instructions received, is presented with, say, the letter B, and required to find that letter in a subsequent array. Identity and category information are activated as a result of the 
presentation of $B$. If the array that follows constitutes a between-category task, field items (digits), unprimed, do not attract a great deal of attention, and identification of the target is relatively rapid. If the task is within-category, field items attract close attention because category information contained within them is primed, and search is relatively slow.

To test the priming hypothesis, a stimulus presumed to act as a prime might be presented, in addition to a target, prior to a search display. Both prime and target would arouse category information. If this should match, the traditional category effect would be obtained. If it should conflict, a between-category search task would, effectively, become a within-category one, since in both tasks, information contained in field items as well as the target would attract close attention. The category effect would be nullified.

Such a procedure was instituted. The stimuli were sketches of male and female faces. Two single faces were presented in succession prior to a search display containing three faces. One of the two faces was intended to act as a prime, and the other was the target (in target-present trials). Stimuli hypothesized to act as primes also acted as potential targets, since the subjects were required to find either of the two faces in the subsequent display.

\section{METHOD}

\section{Subjects}

Sixteen right-handed university students who responded to requests for assistance were paid for their services. Half were female and half were male. One subject was dropped because of a failure to meet an error criterion of $<10 \%$.

\section{Materials}

The stimuli were Letraset Instant Lettering faces AA109 and AA110, and visual patterns $\mathbf{S 7 3 4 0}$ (see the Appendix). Six of the faces were appointed targets, and six were appointed nontargets, with an equal number of males and females in each group. In each of the target and nontarget groups, two faces faced forward, two presented an approximately $3 / 4$ profile to the left, and two an approximately $3 / 4$ profile to the right, these orientations being balanced across sex. Stimuli were back-projected as $35-\mathrm{mm}$ positive slides onto a $13 \times 13 \mathrm{~cm}$ screen. At a viewing distance of $1 \mathrm{~m}$, a display of three faces subtended a horizontal visual angle of $4.6^{\circ}$ and a vertical angle of $5.7^{\circ}$. At the same distance, a display containing a single face subtended a horizontal angle of $2.4^{\circ}$ and a vertical angle of $3.1^{\circ}$, and a display containing a visual pattern subtended visual angles of $1.5^{\circ}$.

The stimuli formed sets of three slides. The first two slides, comprising a memory set, each contained a single face. The third slide contained a search display of three faces. In each of 24 mixed-sex search displays, a male or a female target appeared in one corner of an imaginary upright or inverted triangle at random, with the constraint that each of the six target faces appear in each position of the triangle no more than twice. The two remaining positions were filled at random with two different nontarget faces of a sex opposite that of the target face, with the constraint that each of the six nontarget faces appear an equal number of times. This pattern was repeated in each of 24 same-sex search displays on target-present trials, except that now the two nontarget faces were of the same sex as was the target face. In each of 24 search displays in all target-absent trials, three different nontarget faces of the same sex appeared at random, one in each of the three corners of an imaginary triangle, with the constraint that each face appear an equal number of times.
Projected in the same tachistoscopic field, the stimuli in all three slides were not overlaid exactly, in order to avoid masking effects. The targets in the search displays were left-right reversed to reduce gross physical feature overlap between these stimuli and those in the first and second slides.

\section{Design}

The first two slides in the sets of three bore three kinds of relationships to each other. In the neutral condition, one of the slides contained a face and one contained a visual pattern. Serial positions of face and pattern were varied at random, as was presentation of the three kinds of patterns. In the same condition, each of the two slides contained a single face of the same sex as that contained in the other. In the different condition, each of the two slides contained a single face of a sex opposite that contained in the other. In all conditions, a face in the first or second slide was male half the time, female half the time, a target half the time and, supposedly, a prime half the time (except in the neutral condition, from which primes were absent). Half of the time, one of the three faces in the third slide was either the face presented in the first or the face presented in the second slide (or in the neutral condition, it was simply the lone face in the first or second slide).

The three factors of type of search (between-category, withincategory), type of target (neutral, same, different), and serial position of target (first, second) were within-subjects factors in a $2 \times 3 \times 2$ design.

The neutral, same, and different conditions were each blocked over 16 trials. Between-category and within-category search trials were randomized, with the constraint that an equal number of each appear within each block. There were equal numbers of target-present and target-absent trials for between-category and for within-category search tasks within each block.

Target-present and -absent search displays, as well as within-category and between-category target-present search displays, were counterbalanced over two sets of 48 trials. The two sets were presented in alternate order to each subject six times, with the order of presentation (which set came first) randomized.

\section{Procedure}

The subject was seated at a screen in a dimly lit room, facing two response keys. Tasks relating to the three conditions (neutral, same, different) were explained, and the subject was instructed to respond, by pressing the key appropriate to the favored hand, if a face in the third slide was recognized from the two previous slides. This face might be male or female, would be a reversed image, and might appear with two same-sex or opposite-sex faces. Recognition would be warranted $50 \%$ of the time at random intervals. Otherwise, no response was to be given. Initially, three blank slides would signal the approach of a cue trial, in which first and second slides revealed what kind of task would follow. In cue trials, the third slide was omitted, and no response was required.

The first and second stimuli in the sets were each presented for $500 \mathrm{msec}$ and each followed by an interval of $500 \mathrm{msec}$. The third stimulus was presented for $2 \mathrm{sec}$ and was followed by an interval of $4 \mathrm{sec}$.

The experimenter stood by the subject during the first 48 trials, encouraging fast and especially accurate responding. These trials were treated as practice and were not included in the analysis. Following practice trials, no feedback for responding was given. The subjects had a short rest part of the way through the experiment.

\section{RESULTS}

\section{Reaction Times}

A three-factor analysis of variance was performed on the mean correct reaction times (RTs) for each subject. Significant main effects were found for each of the three factors of search condition, type of target, and serial position of target. Between-category search RTs were faster than within-category search RTs $[F(1,15)=10.48, p<$ $.01]$. The mean RT for between-category search was 
$758 \mathrm{msec}$, and for within-category search, $793 \mathrm{msec}$. Reliable differences were found among the RTs of the neutral, same, and different conditions $[F(2,30)=92.3$, $p<.001]$. Separate analyses conducted to elucidate these differences showed that RTs in the neutral condition were faster than RTs in the same condition $[t(15)=8.97$, $p<.001$ ], and that RTs in the same condition were faster than RTs in the different condition $[t(15)=3.84, p<.01]$. These effects can be explained in terms of the level of difficulty involved in a given task, and they were most notable between RTs in the neutral condition, in which only one target face was sought, and RTs in both the same and the different conditions, in which a target face was sought that might be either of two faces presented. Mean RTs were $668 \mathrm{msec}$ in the neutral condition, $811 \mathrm{msec}$ in the same condition, and $849 \mathrm{msec}$ in the different condition. RTs when targets were in Serial Position 2 (mean RT, $758 \mathrm{msec}$ ) were faster than RTs when targets were in Serial Position 1 (mean RT, $793 \mathrm{msec}$ ) $[F(1,15)=$ $5.26, p<.05]$. Although this result only just reached significance, it may be a reliable effect attributable to the preempting, by the target in Position 2, of the memory comparison processes that occur when the search display is presented; or it may be that the target in Position 2 possesses a stronger memory trace.

The critical result is illustrated in Figure 1. This was the interaction observed between search condition and type of target $[F(2,30)=23.8, p<.001]$. More specific analyses of this interaction revealed that in the neutral con-

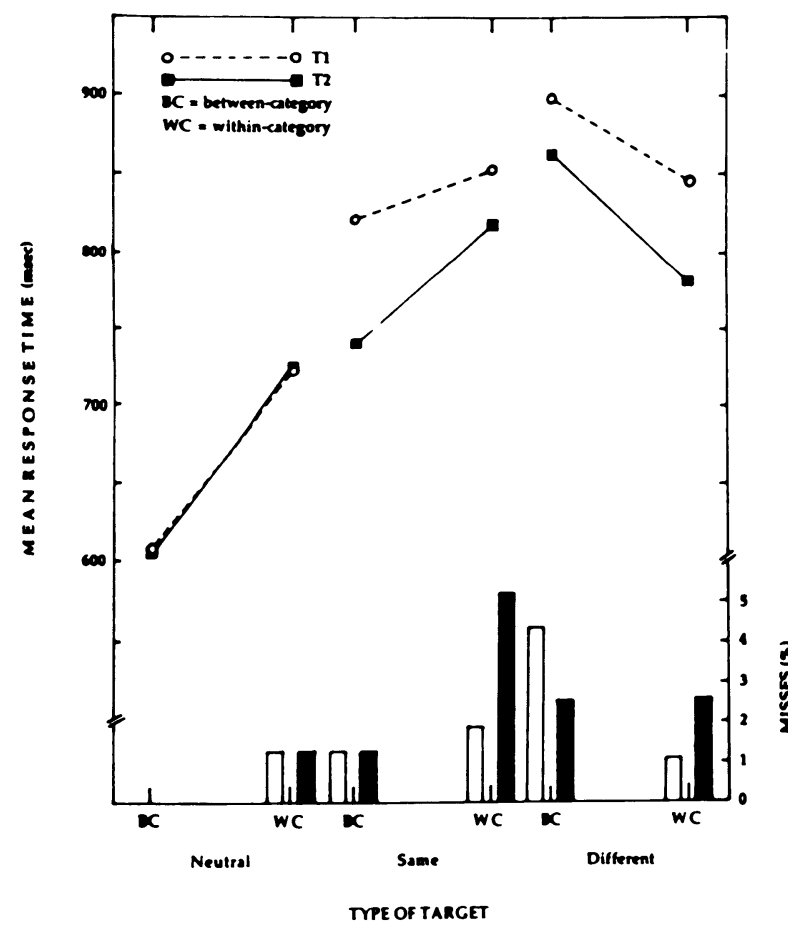

Figure 1. Mean reaction times and percentage of errors of omission (misses) as a function of between-category or within-category search, type of target, and serial position of target (T1,T2). dition, RTs in between-category trials were faster than RTs in within-category trials $[t(15)=8.02, p<.001]$, and that in the same condition, RTs in between-category trials were faster than RTs in within-category trials $[t(15)=2.65, p<.01]$. This pattern was reversed in the different condition, in which RTs in between-category trials were slower than RTs in within-category trials $[t(15)=3.3, p<.01]$. No other significant interactions were found.

\section{Errors}

Errors consisted of $0.4 \%$ false alarms ("yes" responses on target-absent trials), and $1 \%$ misses (the absence of "yes" responses on target-present trials). The distribution of misses over conditions is illustrated in Figure 1. In general, it followed the pattern found for RTs, suggesting that subjects were not trading speed for accuracy.

\section{DISCUSSION}

The traditional category effect was observed both when visual noise and a target face were presented in succession prior to search displays and when two same-sex faces were presented in succession prior to search displays. In each case, search was reliably faster with mixed-sex than with same-sex faces. When two opposite-sex faces were presented in succession prior to search displays, the category effect was not only nullified, but reversed. Search was now reliably faster with same-sex than with mixed-sex faces.

Both traditional and reversed category effects can be explained plausibly with reference to a priming account. First, assume for argument's sake that one nontarget face in a search display is usually encountered prior to recognition of a target face, and that effects are averaged over target position. Assume further there is an expectation that a stimulus presented will assist in a subsequent search task one times out of two and that category information contained within it will be relevant. The stimulus will be encoded, and pertinent identity and category information will be extracted, stored, and maintained in a state of activation as a consequence of the operation of priming processes (as well as mental effort that the subject makes). A conditional tendency to respond will also be activated in expectation of the response task ahead.

Consider a target-present trial in which memory set faces are both female. Category information is duplicated and the tendency to respond to a female face will be strong, although it is spread over two identities. If the search task is mixed-sex, information contained in a male face in the search display, when matched against the information activated by the memory set, will be seen to be irrelevant with respect to identity as well as category, and it will be examined no further. It will arouse a strong tendency in the subject not to respond (a "no-go" response). When material activated by the female face in the search display is matched against that activated by the memory set, a strong category and identity match will be obtained with one memory set face as a consequence of perceptual and category priming processes. The female target face in the search display will elicit a strong response tendency (a "go" response). This tendency will be inhibited somewhat by the tendency derived from the category match effected between the target in the search display and the potential-target memory set face, but it will be distinct from the tendency aroused by the male search display face. The response performed will thus be rapid. But if two female faces are presented prior to a same-sex display, strong conflicting response tendencies will be aroused as a result of the match between category information activated by the memory set and nontarget faces on the one hand, and the memory set and target face on the other. The response will be relatively slow.

Consider next a target-present trial in which memory set faces are mixed-sex. Two kinds of category information will be stored and activated as well as two identities. "Go" response tendencies aroused will be spread over all four entities, and will therefore be only moderately 
strong. If the search task is also mixed-sex, the situation will be complex. Conflicting tendencies will be activated with respect to male as well as female category information contained in the search display, a confusing situation that will cause considerably slowed responses. If a mixed-sex memory set is followed by a same-sex search display, there may be less competition from response tendencies aroused by misleading category information than when a same-sex display is preceded by same-sex faces. Reaction time may not be faster, however, because when memory set faces are mixed-sex, the response activated will be driven by an only moderately strong impulse.

The results of this study are perhaps particularly interesting with respect to the faster within-category search observed in the different condition, a finding that any other explanation for the category effect cannot, apparently, accommodate with ease. Faster within-category search has been observed by several researchers, albeit incidentally, and it can readily be explained with reference to a priming account (see, e.g., Proctor \& Fober, 1988; Shaffer \& LaBerge, 1979). Response competition derived from either passive or active category priming, or both, can also account plausibly for other findings in the literature. Examples of these findings are: Dixon and Shedden's (1987) observation of a category effect with alphanumeric stimuli matched both between and within categories; Henderson and Chard's (1978) category effect with semantic stimuli; the "oh-zero" phenomenon (Jonides \& Gleitman, 1972); certain findings attributed to automatic processing (Palmer \& Jonides, 1988; Shaffer \& LaBerge, 1979); inferior recall of field items in between-category search (Jonides \& Gleitman, 1976); and the "pop-out" effect (Jonides \& Gleitman, 1972).

Despite its post hoc nature, the model outlined above to explain the observed effects offers at least conditional support for a priming account of the category effect. The findings need both corroboration and refinement, however. A major methodological refinement called for is the substitution of a noninformative stimulus - that is, one that never gives any clue of the identity of a target-for a potential target in memory sets. The effects on search rate and accuracy of category information contained within a stimulus irrelevant in search might thus be distinguished, and the priming account given stronger support. Subjects could be instructed to attend to such a stimulus, but told that it would be of no help to them in the forthcoming task. As things stand, there is a confound between passive priming effects and the active effort that a subject makes to remember a stimulus thought perhaps to be relevant in an ensuing task. Using a noninformative stimulus would also preclude any intertrial priming with respect to response assignment, as well as the tendency for conditional responses to be activated: In theory, noninformative primes would arouse only implicit identity and category information.

\section{REFERENCES}

BUtLeR, B. E. (1980). The category effect in visual search: Identification versus localization factors. Canadian Journal of Psychology, 34, 238-247.

Deutsch, J. A. (1977). On the category effect in visual search. Perception \& Psychophysics, 21, 590-592.

Dick, A. O. (1971). Processing time for naming and categorization of letters and numbers. Perception \& Psychophysics, 9(3B), 350-352.

Dixon, P., \& SHEDDEN, J. M. (1987). Conceptual and physical differences in the category effect. Perception \& Psychophysics, 42, 457-464.

DunCaN, J. (1980). The locus of interference in the perception of simultaneous stimuli. Psychological Review, 87, 272-300.

Duncan, J. (1983). Category effects in visual search: A failure to replicate the "oh-zero" phenomenon. Perception \& Psychophysics, 34, 221-232.

FLETCHER, B. (C). (1981). Alphanumeric category effects: Evidence against the sufficiency of a localization explanation. Perception \& Psychophysics, 30, 347-352.

Henderson, L., \& ChARD, J. (1978). Semantic effects in visual word detection with visual similarity controlled. Perception \& Psychophysics, 23, 290-298.

Jonides, J., \& Gleitman, H. (1972). A conceptual category effect in visual search: $\mathrm{O}$ as letter or as digit. Perception \& Psychophysics, 12, 457-460.
Jonides, J., \& Gleitman, H. (1976). The benefit of categorization in visual search: Target location without identification. Perception \& Psychophysics, 20, 289-298.

Kahneman, D., \& Treisman, A. (1984). Changing views of automaticity and attention. In R. Parasuraman \& D. R. Davies (Eds.), $\mathrm{Va}$ rieties of attention (pp. 29-61). New York: Academic Press.

KARLIN, M. B., \& Bower, G. H. (1976). Semantic category effects in visual word search. Perception \& Psychophysics, 19, 417-424.

KRUEGER, L. E. (1984). The category effect in visual search depends on physical rather than conceptual differences. Perception \& Psychophysics, 35, 558-564.

NiCKERSON, R. S. (1973). Can characters be classified directly as digits vs letters or must they be identified first? Memory \& Cognition, 1 , 477-484.

Palmer, J., \& Jonides, J. (1988). Automatic memory search and the effects of information load and irrelevant information. Journal of Experimental Psychology: Learning, Memory, \& Cognition, 14, 136-144.

PoSNER, M. I., \& SNYDER, C. R. R. (1975). Facilitation and inhibition in the processing of signals. In P. M. A. Rabbitt \& S. Dornic (Eds.), Attention \& Performance $V$ (pp. 669-682). Hillsdale, NJ: Erlbaum.

Proctor, R. W., \& Fober, G. W. (1988). A response-selection basis for the mixed-category, repeated-stimulus inferiority effect. Perception \& Psychophysics, 44, 182-190.

SHAFFER, W. O., \& LABERGE, D. (1979). Automatic semantic processing of unattended words. Journal of Verbal Learning \& Verbal Behavior, 18, 413-426.

WHITE, M. J. (1977). Identification and categorization in visual search. Memory \& Cognition, 5, 648-657.

\section{Appendix}

Stimulus Faces, and Examples of Memory Sets and Search Displays

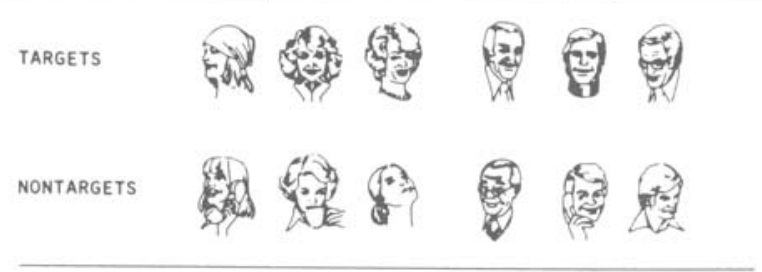

MEMORY SETS

SEARCH DISPLAYS

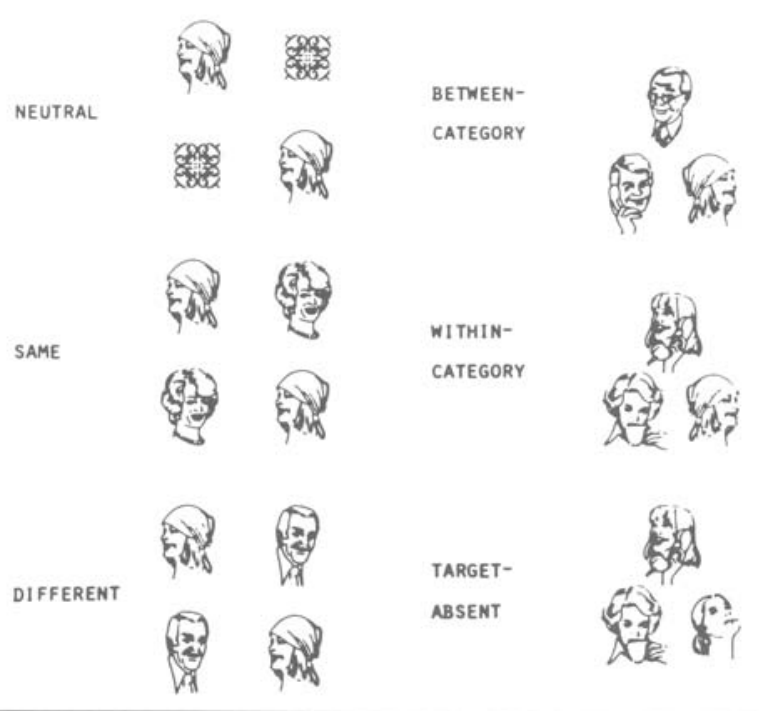

(Manuscript received March 13, 1991.) 